TRANSACTIONS OF THE

AMERICAN MATHEMATICAL SOCIETY

Volume 280, Number 1, November 1983

\title{
SOME RESEARCH PROBLEMS ABOUT ALGEBRAIC DIFFERENTIAL EQUATIONS
}

BY

LEE A. RUBEL ${ }^{1}$

\begin{abstract}
Twenty-four new research problems are posed, and their background and partial solutions are sketched. Many of these problems are in the (somewhat unexpected) area of interaction between algebraic differential equations, topology, and mathematical logic.
\end{abstract}

Introduction. What has struck me most about the subject of algebraic differential equations (ADEs) is how easy it is to pose hard problems. Another way of saying the same thing is that the subject is still in a formative state, with many fundamental problems not even posed, much less solved.

I am going to present the first part (Problems 1-3) of this paper as I first wrote it. There is a rather dramatic twist at its end, as you will see.

Explanatory section. An algebraic differential equation (ADE) is just one where the left-hand side is a polynomial in everything. More specifically, let $P\left(x_{0}, x_{1}, \ldots, x_{n+1}\right)$ be a polynomial in $n+2$ variables, not the zero polynomial, with complex coefficients, or with real coefficients according to the context. Then the equation

$$
P\left(z, w(z), w^{\prime}(z), \ldots, w^{(n)}(z)\right)=0
$$

is called an ADE. We may write this as

$$
P(z, \vec{w})=0 .
$$

In the real case, we prefer to write

$$
P\left(x, y(x), y^{\prime}(x), \ldots, y^{(n)}(x)\right)=0
$$

or just

$$
P(x, \vec{y})=0 .
$$

In both of these contexts, we call $P$ a differential polynomial.

What we mean by a "solution" of an ADE depends somewhat on the context. In the complex case it will mean an analytic (or sometimes a meromorphic) function on a region $\Omega$ that satisfies (1). When this happens, we call $w(z)$ a hypotranscendental function. If $w(z)$ satisfies no $\mathrm{ADE}$, then it is assigned the beautiful adjective transcendentally transcendental. (See [OST] for a good introduction to this subject. In

Received by the editors January 14, 1982.

1980 Mathematics Subject Classification. Primary 34-XX, 34A34.

${ }^{1}$ The research of the author was partially supported by a grant from the National Science Foundation. 
particular, it is shown there (see also [MOO]) that the sum, product, difference, quotient, and composition (under suitable restrictions) of two hypotranscendental functions is again hypotranscendental). Many of the special functions of complex analysis are known to be hypotranscendental. For example, the polynomials, exponential and trigonometric functions and their inverses, Bessel functions, Hermite functions, etc., are all hypotranscendental. On the other hand, $\Gamma(z)$ (the Euler gamma function) and $\zeta(z)$ (the Riemann zeta function) are known to be transcendentally transcendental. (See [BAK and OST], respectively, for proofs of these classical facts.)

Apart from their intrinsic appeal and many applications, algebraic differential equations have a special importance because of their close relation with analog computers (see [SHA and POE] for details).

Still in the complex domain, there is another kind of object, namely a formal power series (fps) that can be a candidate for a solution of an ADE. By fps we mean a series $f(z)=\sum_{n=0}^{\infty} a_{n} z^{n}$, where no convergence of any kind is required. Thus, $\sum n ! z^{n}$ is an fps. Via the usual definitions, two fps $f(z)$ and $g(z)$ may be added and multiplied. Also, an fps $f(z)$ may be differentiated term by term to get another fps $f^{\prime}(z)$. So it makes sense, if $P$ is a differential polynomial, to write

$$
P\left(z, f(z), f^{\prime}(z), \ldots, f^{(n)}(z)\right)=0,
$$

and if it is actually true, then we also call $f(z)$ a solution of the ADE (3). Several of our problems are best posed in this more general context. An extensive treatment of fps that satisfy an ADE (found in [POP and OST]) also bears directly on this aspect. My favorite result in this area is the theorem of Maillet [MAI] (see also [MAH2]) that if $\sum a_{n} z^{n}$ satisfies an $\mathrm{ADE}$, then there is a constant $\alpha$ so that $\left|a_{n}\right| \leqslant K(n !)^{\alpha}$ for all $n$ and some constant $K$.

In the real domain, the best results on solutions of (2) are obtained if it is assumed that $y(x)$ is in $C^{\infty}$ (i.e., infinitely differentiable) or is analytic. However, note that (2) makes sense if we only assume that $y(x) \in C^{n}$, or even $y(x) \in D^{n}$, which means that $y(x)$ is $n$ times differentiable. It is shown in [RUB2] that many of the classical results for the $C^{\infty}$ case fail completely for the $C^{n}$ case. This is important to bear in mind when reading the classical literature, where the meaning of the word "solution" is not emphasized. In [RUB2], the definition is proposed that we call a function $y(x)$ a solution of (2) on an open interval $I$ provided that (i) $y(x)$ is continuous on $I$, (ii) $y(x)$ is analytic on a dense open subset $\Omega$ of $I$, and (iii) $y(x)$ satisfies (2) on $\Omega$. We will not go further into these matters here. An exhaustive treatment was made of certain algebraic aspects of the $C^{\infty}$ and analytic cases by Ritt and his school (see [RIT1, RIT2, KOL and KAP]).

We conclude this explanatory section with a little terminology. If $P$ involves $x_{n+1}$ (i.e. $\left.w^{(n)}\right)$ in a nontrivial way, then we say that (1) has order $n$. We say that one differential polynomial $P$ is lower than another one $Q$ if either (i) $P$ is of lower order than $Q$ or (ii) $P$ is of the same order as $Q$, but of lower degree than $Q$ in $w^{(n)}$. We note that, given any property that some differential polynomial has, there is a lowest one with this property (it need not be unique). Finally, if $P$ is a differential polynomial, its separant $S=S_{P}$ is defined as $S=\partial P / \partial w^{(n)}$. 
The Problems. The first question is "what is the topological type of the Riemann surface of a solution of an ADE?" Before we expand on this, let us consider some related problems.

Problem 1. Can an ADE have a solution which has the unit circle as a natural boundary?

REMARK 1. I expect that the answer is no, in which case one would have an alternative proof that the function $\sum z^{2^{n}}$ satisfies no ADE (see [MAH1]). It seems likely that $\theta(z)=\sum_{n=0}^{\infty} z^{n^{2}}$ satisfies no ADE.

Problem 2. What kind of gaps can the power series of a solution of an ADE have?

REMARK 2. If by solution we mean an analytic function rather than merely a formal power series, then this is related to Problem 1, since a function with radius of convergence 1 cannot have Hadamard gaps or even Fabry gaps in its Taylor series unless it has the unit circle as natural boundary. Here, we write $f(z)=\sum a_{k} z^{n_{k}}$, and say that $f(z)$ has Hadamard gaps if $\lim \inf n_{k+1} / n_{k}>1$ and Fabry gaps if $\lim n_{k} / k$ $=\infty$. Popken in [POP] (see also [MAH3]) has shown that really huge gaps ("Hadamard gaps of ratio $\infty$ ") are not possible, i.e., if $\lim n_{k+1} / n_{k}=\infty$, and $f$ satisfies an $\mathrm{ADE}$, then $f$ is a polynomial.

Problem 3. If a formal power series $f(z)=\sum a_{n} z^{n}$ has only a finite set of different coefficients (i.e. $\left\{a_{n}: n=0,1,2, \ldots\right\}$ is finite) and if $f$ satisfies an $A D E$, must $f$ be $a$ rational function?

REMARK 3. The affirmative answer would follow from the expected negative answer to Problem 1 (and would strengthen our anticipation of it), because of the classical theorem of Polya [DIE] that a power series with finitely many different coefficients either has the unit circle as natural boundary, or is a rational function.

Dramatic twist. I had been worrying about these problems for several years, when one morning, I decided to write the present exposition. Later that morning, after the writing, I went to the library for a breather, and was browsing through the bibliography in [XXX] of Jules Drach, when the reference [DRA] leapt out at me. I hurried to consult it, and found the more primary reference [JAC], where in 1847, Jacobi solved some problems I was posing in 1981! And I found it less than an hour after writing down the problems.

But as Drach says "La complication de cette équation a sans doute entrainé sa disparition des Ouvrages classiques, ou même des Mémoires sur les fonctions modulaires." Nevertheless, even though Drach found in [DRA] a much simpler way of expressing Jacobi's differential equation, the result is still very far from being well known.

THEOREM (JACOBI 1847). The function $\theta_{3}(\tau)=1+2 q+2 q^{4}+2 q^{9}+\cdots$, where $q=e^{i \pi \tau}$, regarded as depending on the variable $\rho=\log q=i \pi \tau$, satisfies the third-order algebraic differential equation

$$
\left(y^{2} y^{\prime \prime \prime}-15 y y^{\prime} y^{\prime \prime}+30 y^{\prime 3}\right)^{2}+32\left(y y^{\prime \prime}-3 y^{\prime 2}\right)^{3}=y^{10}\left(y y^{\prime \prime}-3 y^{\prime 2}\right),
$$

where the accents indicate derivations with respect to $\rho$. 
Notice that this result answers (at least partially) Problems 1-3 at a single stroke. For, after some obvious changes of variable, and writing (nonstandard notations) $\theta(z)=\sum_{n=0}^{\infty} z^{n^{2}}$, we see that $\theta(z)$ does satisfy an ADE, yet it has the unit circle as natural boundary, has Fabry gaps in its power series, and has only two different Taylor coefficients. The proofs of Jacobi's theorem use special properties of elliptic functions, so that the following problem seems open.

Problem 4. Let $\theta_{p}(z)=\sum_{n=0}^{\infty} z^{n^{p}}$. For which values of $p$ does $\theta_{p}$ satisfy an $A D E$ ?

Problem 5. For which regions $\Omega$ in the plane does there exist a hypotranscendental function $\theta_{\Omega}$ that has $\Omega$ as natural boundary?

The remaining problems. To formulate the main problem about topological type, we need some preliminaries. If $f(z)=\sum a_{n}\left(z-z_{0}\right)^{n}$ satisfies an ADE and has a positive radius of convergence, we can follow Weierstrass and construct its analytische Gebilde, that is, the open Riemann surface of all analytic continuations of $f$. According to a theorem of Stoilow-Kerekjarto (see also [RIC]), every open Riemann surface is homeomorphic to a sphere with a countable number of handles-the bases of the handles accumulating on the equator of the sphere-after a closed point set is removed from the equator.

Problem 6. What is the topological type of the Riemann surface of a hypotranscendental function?

That is, what can be the set of handles and the set removed from the equator in the Stoilow-Kerekjarto representation?

REMARK 6. Notice that when $f$ is algebraic (so that the ADE is of order 0 , namely $P(z, f(z))=0$ ) then a celebrated theorem of Riemann says that then (and only then) the Riemann surface is compact.

Problem 7. Can the deleted set be a Cantor set?

REMARK 7. This would seem very unlikely. Note that Jacobi's theorem shows that the deleted set can be a segment. There is a theorem of Painlevé [PAI] about first order equations with solutions that have a "line of singularities". In this direction, we prove the following result.

THEOREM. Let $\gamma$ be an arc in $\mathrm{C}$ and let $f(z)$ be analytic on one side of $\gamma$ so that for every $k=0,1,2, \ldots$ and for every $z_{0} \in \gamma, \lim _{z \rightarrow z_{0}} f^{(k)}(z)$ exists, where we understand this to be a one-sided limit. If $f(z)$ is hypotranscendental, then $f(z)$ is analytically continuable across some subarc of $\gamma$.

Proof. Suppose $f(z)$ fulfills our hypotheses. Ordering the differential polynomials as we described earlier, there must be a lowest $\operatorname{ADE}, P(z, \vec{f}(z))=0$ satisfied by $f(z)$.

At a point $z_{0}$ on $\gamma$, we write $f^{(l)}\left(z_{0}\right)=\lim _{z \rightarrow z_{0}} f^{(l)}(z)$ for $l=0,1,2, \ldots$ Then $f$ satisfies $P\left(z_{0}, \vec{f}\left(z_{0}\right)\right)=0$ on $\gamma$. At a point $z_{0}$ on $\gamma$ where the separant $\partial P / \partial f^{(n)} \neq 0$, we can solve $P(z, \vec{f}(z))=0$ for $f^{(n)}(z)$ in normal form

$$
f^{(n)}(z)=\alpha\left(z, f(z), f^{\prime}(z), \ldots, f^{(n-1)}(z)\right),
$$


by using the implicit function theorem, where $\alpha$ is an analytic algebraic function of its arguments in a neighborhood of $\left(z_{0}, f\left(z_{0}\right), \ldots, f^{(n-1)}\left(z_{0}\right)\right)$. By a basic existence theorem (see [HIL]) for solutions of ODEs in the complex domain it follows that $f$ has an analytic extension to a neighborhood of $z_{0}$. If, therefore, $\gamma$ is a line of singularities of $f(z)$, we must have $S=\partial P\left(z_{0}\right) / \partial f^{(n)}=0$ on a dense set of $z_{0}$ on $\gamma$, and hence for every $z_{0} \in \gamma$. It follows that $S$ vanishes identically in a one-sided neighborhood $\Omega$ of $\gamma$ because $f$ is analytic there. But the equation $\partial P / \partial f^{(n)}=0$ is a lower $\mathrm{ADE}$ than the original one, and this contradiction proves the theorem.

Problem 8. If $w(z)=\sum_{n=0}^{\infty} a_{n} z^{n}$ satisfies an $A D E P(z, \vec{w}(z))=0$, must there exist another $A D E Q(z, \vec{s}(z))=0$ that is satisfied by every partial sum $s(z)=s_{n}(z)=$ $\sum_{k=0}^{n} a_{k} z^{k}$ ?

REMARK 8. We show that the answer is yes in case $y(z)=(1-z)^{-1}$ or $y(z)=\exp z$. First, for $y(z)=(1-z)^{-1}, s_{n}(z)=\left(1-z^{n+1}\right) /(1-z)$. Let $s(z)=s_{n}(z)$. Then $s(z)(1-z)=1-z^{n+1}$. Now $z^{n+1}$ satisfies the $\operatorname{ADE}\left(z w^{\prime} / w\right)^{\prime}=0$, and 1 , of course, satisfies an ADE (that is independent of $n$ ) (see [MOO or OST]), and then by the same references, so must $s(z)$. For $\exp z$, with $s(z)=s_{n}(z)$, we have $s_{n}(z)-s_{n}^{\prime}(z)=z^{n} / n !$, and if we let $g(z)=s_{n}(z)-s_{n}^{\prime}(z)$, then we have $\left(z g^{\prime} / g\right)^{\prime}=0$.

Problem 9. Given an open interval $I$, say $I=(0,1)$, or $I=(-\infty, \infty)$, is there an algorithm for deciding whether the $A D E P(x, \vec{y})=0$, where $P$ has integer coefficients, has a global solution y on I?

REMARK 9. Possibly, the answer may be different depending on the restrictions on $y$, i.e. whether $y$ is analytic, $C^{\infty}, C^{n}$ or merely $D^{n}$. In [RUB2] an example was given of an ADE on a certain interval $I$ that has $C^{\infty}$ solutions, but no analytic solutions. To get a little of the flavor of the problem, look at the equation $y^{\prime}-\left(y^{2}+1\right)=0$, which has a global solution on $I$ precisely when the length of $I$ does not exceed $\pi$. If I had to guess, I would guess that no algorithm of the kind asked for actually exists. (Added later. The $C^{\infty}$ case of this problem has just been settled by J. Denef and L. Lipshitz, in the expected negative direction, in a 1982 preprint.)

Problem 10. Let $G$ be a simply connected region, but $G \neq \mathbf{C}$, and suppose that every conformal selfmap of $G$ onto $G$ satisfies an $A D E$. Musi $G$ be conformally equivalent to the unit disc $\mathbf{D}$ via a map $\phi$ that satisifes an $A D E$ ?

To prepare the way for the next problem, we shall define the Ahlfors function. If $G$ is a (presumably multiply connected) region and $z_{0}$ is a point in $G$, we define the Ahlfors function $\alpha_{z_{0}}$ with base point $z_{0}$ as the (unique) solution of the following extremal problem: (i) $\alpha\left(z_{0}\right)=0$, (ii) $|\alpha(z)| \leqslant 1$ for all $z \in G$, (iii) $\alpha^{\prime}\left(z_{0}\right)$ is as large as it can be for the class of functions satisfying (i) and (ii). In case $G$ is simply connected, $\alpha_{z_{0}}$ becomes the Riemann map of $G$ onto $\mathbf{D}$ that takes $z_{0}$ to 0 , with positive derivative there.

Problem 11. Suppose $\alpha_{z_{0}}$ is hypotranscendental, and let $z_{1} \in G$ be another base point. Must $\alpha_{z_{1}}$ be hypotranscendental too? 
REMARK 11. Note that the answer is affirmative if $G$ is simply connected, since the conformal one-one maps of $\mathbf{D}$ onto $\mathbf{D}$ are the Möbius transformations, which are rational functions.

We now step into the class of hypoalgebraic differential equations (HPE?). Consider the class of functions $H$ of $n+2$ variables that are built up from the positive real numbers and the real variables $x_{0}, x_{1}, \ldots, x_{n+1}$ by addition $(f+g)$, multiplication $(f \cdot g)$ and two-place exponentiation $\left(f^{g}\right)$. Consider the differential equation

$$
H_{1}\left(x, y(x), y^{\prime}(x), \ldots, y^{(n)}(x)\right)=H_{2}\left(x, y(x), y^{\prime}(x), \ldots, y^{(n)}(x)\right) .
$$

We shall call it a hypoalgebraic differential equation (see [TAR and HER] for related discussions). One example might be

$$
y^{\prime}(x)^{y(x)^{\nu^{\prime \prime}(x)}}+\left(3 x^{2}+1\right)^{y^{\prime}(x)}=x^{2}+y(x) y^{\prime}(x) .
$$

(If this equation upsets you, please skip this problem.)

Problem 12. If y satisfies a hypoalgebraic differential equation, must $y$ then satisfy an $A D E$ ?

RemarK 12. I think the answer is yes in case $y \in C^{\infty}$ by taking numerous derivatives and then eliminating (using resultants) the towers and also the logarithms that creep in.

For the next problem, notice that the equation $y^{\prime 2}=4 y$ has the solution

$$
\begin{cases}y=0 & \text { for } x \leqslant a, \\ y=(x-a)^{2} & \text { for } x>a,\end{cases}
$$

which has a break in the second derivative at $x=a$. Also note that for the initial condition $y(a)=0$, the ADE has more than one solution, e.g. $y \equiv 0$ and $y \equiv$ $(x-a)^{2}$. The next question asks, for a general ADE in the real domain, whether having a solution $y_{0}$, with a break in some derivative implies having more than one solution with the same initial conditions as $y_{0}$ at the breaking point.

Problem 13. Let $P(x, y)=0$ he an $A D E$ that has $y_{0}$ as a pointwise solution (i.e. $y_{0}$ is $n$-times differentiable and satisfies $P(x, \vec{y})=0)$. Suppose $y_{0}^{(k)}(x)$ has a discontinuity at $x=x_{0}$, but is continuous in a deleted neighborhood of $x_{0}$. In some smaller neighborhood of $x_{0}$ must there exist a second solution $y_{1} \neq y_{0}$ but such that $y_{1}\left(x_{0}\right)=$ $y_{0}\left(x_{0}\right), y_{1}^{\prime}\left(x_{0}\right)=y_{0}^{\prime}\left(x_{0}\right), \ldots, y_{1}^{(n)}\left(x_{0}\right)=y_{0}^{(n)}\left(x_{0}\right)$, where $n+1$ is the order of $P$ ?

By ZFC, we mean the Zermelo-Fraenkel axioms for set theory together with the axiom of choice (see [TAZ]). Up till now, at least, ZFC has been an adequate framework for mathematical proofs.

Problem 14. Does there exist an ADE, say with integer coefficients, that has a solution, but that has no solution that is definable in ZFC? How about having no computable solution?

Remark 14. See [ABE and PER] for some related questions. Note that if the equation is first-order and in normal form $\left(y^{\prime}=f(x, y)\right)$, then Wolfgang Walter (see [WAL]) has shown that there is a definable solution, even without the hypothesis 
that the differential equation be algebraic. However, his solution need not be computable (see [ABE and PER] for precise definitions of this word), since it is based on finding the maximum of a continuous function on a closed rectangle. Of course it can be proved in ZFC that this maximum exists, but there is no effective procedure for computing it.

(Added later. In a private communication, M. Magidor has outlined to the author a proof that every $\mathrm{ADE}$ with integer coefficients that has a solution must have a definable solution.)

Problem 15. Is there an ordinary differential equation $F\left(x, y, y^{\prime}, \ldots, y^{(n)}\right)=0$, where $F$ is definable (or even computable) in ZFC, that has solutions, but such that none of them is definable in $\mathrm{ZFC}$ ?

In another direction, the Skolem-Mahler-Lech theorem (see [LEC]) says that if $\sum a_{n} z^{n}=P(z) / Q(z)$ is the quotient of two polynomials, then $Z=\left\{n: a_{n}=0\right\}$ must be a finite union of arithmetic progressions, give or take a finite set. (A fascinating fact about this theorem is that despite its completely elementary statement, the only known proofs depend upon $p$-adic analysis.)

Problem 16. Let $y(z)=\sum a_{n} z^{n}$ be a solution of a linear differential equation,

$$
P_{n}(z) y^{(n)}(z)+\cdots+P_{1}(z) y^{\prime}(z)+P_{0}(z) y(z)=Q(z),
$$

where the coefficients $P_{j}(z)$ are polynomials, and $Q(z)$ is a polynomial. Let $Z=\{n$ : $\left.a_{n}=0\right\}$. Characterize the sets $Z$ that can arise in this way. Are they different from the Skolem-Mahler-Lech case $n=0$ ?

Remark 16. Even the case $n=1$ seems to be challenging. My student, Vichian Laohakosol, is looking at this case, but has no definitive results yet.

Problem 17. Let $y(z)=\sum a_{n} x^{n}$ be a solution of an $A D E$, and as before let $Z=\{n$ : $\left.a_{n}=0\right\}$. Characterize the sets $Z$ of integers that can arise in this way.

The next problems go in different directions.

Problem 18. Consider the linear differential equation

$$
P_{n}(x) y^{(n)}(x)+\cdots+P_{1}(x) y^{\prime}(x)+P_{0}(x) y(x)=Q(x),
$$

where the $P$ 's and $Q$ are polynomials. Describe those functions $\phi(x)$ which are the uniform limits on all of $\mathbf{R}$ of solutions of (*). What class does $\phi$ run over as we run over the class of all such equations?

Remark 18. If we restrict the $P$ 's to be constants, and $Q$ to be zero, then the class is closely related to the class of almost periodic functions [KAT]. I seem to be making good progress on this problem, by relating it to certain $C^{*}$ Banach algebras.

Problem 19. Does there exist an $A D E P(x, \vec{y}(x))=0$ such that for any continuous function $\phi$ on $\mathbf{R}$, and for every constant $\varepsilon>0$, there exists a real-analytic solution $y(x)$ on $\mathbf{R}$ of $P(x, \vec{y}(x))=0$, such that $|y(x)-\phi(x)|<\varepsilon$ for all $x \in \mathbf{R}$ ? 
REMARK 19. The analogue of this, where we only require that $y \in C^{\infty}$ instead of being analytic, has been proved in [RUB1]. For algebraic partial differential equations on the unit cube in $\mathbf{R}^{n}$, Buck has actually found an equation where the polynomial solutions do the approximating (see [BUC]).

Problem 20. Let $f$ be a hypotranscendental entire function, and let $Z(f)=\{z \in \mathbf{C}$ : $f(z)=0\}$ (counting multiplicities). What can you say about $Z(f)$ ?

REMARK 20. Steven Bank in [BAN] has considered the question whether $Z(f)$ may be the set $\mathbf{N}$ of positive integers. So far as I know, nobody has yet produced a sequence $z_{n} \rightarrow \infty$ so that $\left\{z_{n}\right\}$ is not equal to $Z(f)$ for any hypotranscendental entire function $f$.

Problem 21. For what pairs $\left(z_{k}\right),\left(w_{k}\right), k=1,2,3, \ldots$, of sequences of complex numbers does there exist a hypotranscendental entire function $f$ such that $f\left(z_{k}\right)=w_{k}$ for $k=1,2,3, \ldots$ ?

For the next problem we define a partial ordering $\leqslant$ on all entire functions.

DEFINITION. We write $f \leqslant g$ for entire functions $f$ and $g$ if every ADE satisfied by $g$ is also satisfied by $f$.

Problem 22. Discuss the ordering $\leqslant$, even on the exponential polynomials $\sum_{k=1}^{n} a_{k} e^{\lambda_{k} z}$. Do the entire functions with this ordering form a lattice?

REMARK 22. To illustrate the ordering, if only in a simple way, we prove that $k e^{z} \leqslant e^{z}$ for all constants $k$. For suppose that $P(\overrightarrow{z, \exp z})=0$, where we now write $P(z, \vec{y})=\sum_{m=0}^{m=M} z^{m} P_{m}(\vec{y})$, where $P_{m}(\vec{y})$ is autonomous. Now it is easy to prove that for each $m, P_{m}(\overrightarrow{\exp z})=0$. But these are autonomous equations, so $P_{m}(\overrightarrow{\exp (z+\tau)})$ $=0$. Hence, $P_{m}(\overrightarrow{k \exp z})=0$ for each $m$, and the result follows for $k \neq 0$. The case $k=0$ follows by continuity.

Problem 23. Let us now consider a formal power series $m(z)=\sum \mu_{n} Z^{n}$, and call it a multiplier if $m * a$ satisfies an $A D E$ whenever $a(z)=\sum \alpha_{n} Z^{n}$ satisfies an $A D E$, where $m * a(z)=\sum \mu_{n} \alpha_{n} Z^{n}$. The problem is to characterize the multiplies.

REMARK 23. It is easy to see that if $\mu_{n}$ is a rational function of $n$, then $\Sigma \mu_{n} z^{n}$ is a multiplier. Also, if $m(z)$ is a multiplier, then $m(z)$ satisfies an ADE. Could the converse implication be true?

There is a class of "elimination theorems" for ADEs, whose conclusion is that a system $\Sigma$ of ADEs has a differentiability algebraic solution, that is, one in which each of the functions involved satisfies an ADE that involves none of the other functions (see [DEL, RUB3 and SEI]). Of course, some hypotheses are necessary to rule out the possible inconsistency of $\Sigma$.

Problem 24. Let $\Sigma$ be a system of ADEs in finitely many dependent variables that has $a C^{\vec{N}}$ solution on the unit interval $[0,1]$. Is there then some open interval I on which $\Sigma$ has a differentially algebraic solution?

(Note. We use the notation $C^{\vec{N}}$ for a solution to mean that the functions involved are at least as many times continuously differentiable as the system demands. If the 
answer to Problem 24 is affirmative, it would follow from [RUB1] that $\Sigma$ has a solution that is analytic on some interval.)

In summary, these are some aspects of the subject as I have expressed them in problems. I hope not too many of them turn out to be embarrassingly easy. At any rate, I have had hard going making limited progress on a few. I hope people who find complete or partial solutions will communicate them to me and to others. Finally, I hope I have shown that, just a little off the beaten track in differential equations, there lies a fascinating vein of mathematics.

\section{REFERENCES}

[ABE] Oliver Aberth, Computable analysis, McGraw-Hill, New York, 1980.

[BAK] Steven B. Bank and Robert P. Kaufman, A note on Hölder's theorem concerning the gamma function, Math. Ann. 232 (1978), 115-120.

[BAN] Steven B. Bank, Some results on the gamma function and other hypertranscendental functions, Proc. Roy. Soc. Edinburgh Sect. A 79 (1977/78), 335-341.

[BUC] R. C. Buck, The solutions to a smooth PDE can be dense in $C[I]$, J. Differential Equations 41 (1981), 229-238.

[DEL] J. Denef and L. Lipschitz, Power series solutions of algebraic differential equations, preprint, 1982.

[DIE] Paul Dienes, The Taylor series, Dover, New York, 1957.

[DRA] Jules Drach, Sur l'équation différentielle du troisième ordre des fonctions $\vartheta$ elliptiques, C. R. Acad. Sci. Paris Ser. A 206 (1938), 1421-1424.

[HAR] P. Hartman, Ordinary differential equations, Wiley-Interscience, New York, 1969.

[HER] C. Ward Henson and Lee A. Rubel, A solution to Tarski's "high school algebra problem" for a restricted class of exponentials, preprint, 1982.

[HIL] Einar Hille, Ordinary differential equations in the complex domain, Wiley-Interscience, New York, London, Sydney and Toronto, 1976.

[JAC] C. G. J. Jacobi, Über die Differentialgleichung welche die Reihe $1 \pm 2 q+2 q^{4} \pm 2 q^{9}+$ etc., $2 \sqrt[4]{q}+2 \sqrt[4]{q^{3}}+2 \sqrt[4]{q^{25}}+$ etc. genüge leisten, Crelle J. Reine Angew. Math. 36 (1847), 97-112.

[KAP] Irving Kaplansky, An introduction to differential algebra, 2nd ed., Hermann, Paris, 1976.

[KAT] Yitzhak Katznelson, An introduction to harmonic analysis, 2nd ed., Dover, New York, 1976.

[KOL] E. R. Kolchin, Differential algebra and algebraic groups, Academic Press, New York and London, 1973.

[LEC] C. Lech, $A$ note on recurring series, Ark. Mat. 2 (1952), 417-421.

[MAH1] Kurt Mahler, Arithmetische Eigenschaften einer Klasse transzendental transzendenten Funktionen, Math. Z. 32 (1930), 545-585, especially 557.

[MAH2] , On formal power series as integrals of algebraic differential equations, Accad. Naz. Lincei 50 (1971), 76-89.

[MAH3] _ Lectures on transcendental numbers, Springer-Verlag, Berlin and New York, 1976.

[MAI] E. Maillet, Sur les séries divergentes et les équations différentielles, Ann. Sci. Éole Norm. Sup. (3) 20 (1903), 487-518.

[MOO] E. H. Moore, Concerning transcendentally transcendent functions, Math. Ann. 48 (1897), 49-74.

[OST] Alexander Ostrowski, Über Dirichletsche Reihen und algebraische Differentialgleichungen, Math. Z. 8 (1920), 241-298.

[PAI] Paul Painlevé, Mémoire sur les équations différentielles du premier ordre, Oeuvres de Paul Painlevé, Vol. II, CNRS, Paris, 1974, pp. 237-461.

[PER] M. B. Pour-El and Ian Richards, A computable ordinary differential equation which possesses no computable solution, Ann. Math. Logic 17 (1979), 61-90.

[POE] M. B. Pour-El, Abstract computability and its relation to the general purpose analog computer ( some connections between logic, differential equations, and analog computers), Trans. Amer. Math. Soc. 199 (1974), 1-28.

[POP] Jan Popken, Über arithmetische Eigenschaften analytischer Funktionen, North-Holland, Amsterdam, 1935.

[RIC] Ian Richards, On the clasification of noncompact surfaces, Trans. Amer. Math. Soc. 106 (1963), 259-269. 
[RIT1] J. F. Ritt, Differential equations from the algebraic standpoint, Amer. Math. Soc. Colloq. Publ., vol. 14, Amer. Math. Soc., Providence, R.I., 1932.

[RIT2] _ Differential algebra, Amer. Math. Soc. Colloq. Publ., vol. 33, Amer. Math. Soc., Providence, R.I., 1950.

[RUB1] L. A. Rubel, Solutions of algebraic differential equations, J. Differential Equations (to appear).

[RUB2] __, A universal differential equation, Bull. Amer. Math. Soc. (N. S.) 4 (1981), 345-349.

[RUB3] _ An elimination theorem for systems of algebraic differential equations, Houston J. Math. 8 (1982), 289-295.

[SEI] A. Seidenberg, An elimination theory for differential algebra, Calif. Univ. Publ. Math. (N. S.) 3 (1955-1960), 31-65.

[SHA] Claude E. Shannon, Mathematical theory of the differential analyzer, J. Math. Phys. 20 (1941), 347-354.

[TAR] Alfred Tarski, The completeness of elementary algebra and geometry, CNRS, Institut Blaise Pascal, Paris, 1967.

[TAZ] Gaisi Takeuti and Wilson Zaring, Introduction to axiomatic set theory, Springer-Verlag, New York, 1971.

[WAL] Wolfgang Walter, There is an elementary proof of Peano's existence theorem, Amer. Math. Monthly 78 (1971), 170-173.

[XXX] (Author not identified), Eléments pour une étude sur Jules Drach, Cahiers Sem. Histoire Math. 2 (1981), 17-57.

Department of Mathematics, University of Illinois at URbana-Champaign, Urbana, Illinois 61801 